\title{
INFLUÊNCIA DA SUPLEMENTAÇÃO DE CÁLCIO EM MULHERES MENOPAUSADAS NA DOENÇA PERIODONTAL
}

\section{Maria Célia Nunes Pedreira ${ }^{1}$; Teresinha Costa de Santana ${ }^{2}$}

1. Bolsista PROBIC/UEFS, Graduando em odontologia, Universidade Estadual de Feira de Santana, e-mail: mariaceliapedreira@gmail.com

2. Orientadora, Departamento de Saúde, Universidade Estadual de Feira de Santana, e-mail: teresinhasantana@gmail.com

PALAVRAS-CHAVE: Osteoporose; Cálcio; Doenças Periodontais.

\section{INTRODUÇÃO}

Na menopausa ocorre a diminuição da produção e secreção dos hormônios ovarianos devido a um número inadequado de folículos em funcionamento dentro dos ovários ${ }^{1}$. Dentre esses hormônios, está o estrógeno, que é considerado um dos fatores patogênicos dominantes para a osteoporose em mulheres ${ }^{2}$.

A insuficiência estrogênica afeta a capacidade reprodutora feminina, tem ações diretas e indiretas no osso via paratormônio, vitamina D e calcitonina. Pode interferir na absorção de nutrientes, a exemplo do cálcio pelo intestino, na continência urinária, metabolismo ósseo e mineral, na pressão sanguínea e função cardiovascular, nas funções de memória e cognição, bem como na progressão de doenças degenerativas relacionadas com a idade como a osteoporose $^{3}$. Essa patologia é caracterizada pela redução da massa óssea e pela deterioração da micro-arquitetura tanto do osso cortical como trabecular, resultante do desequilíbrio entre a reabsorção e a aposição óssea ${ }^{4}$.

Entendem-se que a Doença Periodontal (DP) é constituída por uma série de processos inflamatórios e infecciosos que atinge os tecidos periodontais, tendo etiologia multifatorial. Essa infecção é induzida por bactérias anaeróbias gram-negativas que colonizam o biofilme dental $^{5}$.

A plausibilidade biológica para a existência dessa associação tem sido sistematizada em torno do seguinte pressuposto: a identificação de receptores de estrógeno no ligamento periodontal sinaliza que a deficiência estrogênica, ao provocar aumento de citocinas reabsortivas, pode promover exacerbação de fatores de inflamação local existentes no periodonto, agravando a condição bucal ${ }^{5,6}$.

Desse modo, pessoas com osteoporose reagiriam a periodontite com aumento local na produção de citocinas e mediadores inflamatórios, como as interleucinas e o fator de necrose tumoral, facilitando a progressão da reabsorção óssea periodontal, ou ainda interferindo nos efeitos da terapia periodontal e dificultando o controle das medidas clínicas periodontais ${ }^{3}$.

Alguns estudos têm mostrado que a suplementação da dieta com cálcio reduz a perda óssea em mulheres na pós-menopausa com baixo consumo desse mineral ${ }^{7}$ e o risco de fraturas em mulheres idosas quando associado com vitamina $\mathrm{D}^{8}$.

Em vista do exposto e da controvérsia sobre o tema, o objetivo deste trabalho foi analisar e comparar dados, já coletados em prontuários, de mulheres menopausadas atendidas pelo NUPPIIM e verificar se existe alguma influência do uso de cálcio no nível de inserção clínica.

\section{MATERIAL E MÉTODOS OU METODOLOGIA (ou equivalente)}

Inicialmente, foi realizado busca sobre o tema nas seguintes bases eletrônicas: the National Library of Medicine, Washington DC, USA (MEDLINE/PubMed), Scientific Electronic Library Online (SciELO), a Literatura Latino-Americana e do Caribe em Ciências da Saúde (LILACS). 
Já a coleta de dados teve como critério de inclusão mulheres pós menopausadas, com ou sem reposição de cálcio. O grupo foi examinado por um pesquisador calibrado do NUPPIIM, que determinou a presença/ausência de osteoporose obtida por exames de mensuração da densidade mineral óssea esquelética. Para avaliar a doença periodontal foram utilizados os índices de profundidade de sondagem, perda de inserção clínica, sangramento gengival. Esta pesquisa foi aprovada pelo Comitê de Ética em Pesquisa da UEFS, sob número de protocolo 199/2009.

Os dados do estudo foram obtidos a partir dos prontuários das participantes e foram organizados em um banco de dados utilizando o programa SPSS17.0,onde foi aplicado o teste ANOVA, com nível de significância em $5 \%$.

\section{RESULTADOS E/OU DISCUSSÃO (ou Análise e discussão dos resultados)}

A população do estudo consistiu em 301 mulheres, faixa etária variando de 50 a 87 anos e média de 60,82 (DP $\pm 7,38$ ) anos, que foram identificadas através das informações sobre idade, idade da menarca, idade da menopausa, filhos nascidos, renda familiar, nível de escolaridade, número de moradores na casa, quantidades de dentes presentes, profundidade de sondagem e nível de inserção clínica. $\mathrm{O}$ exame de densitometria óssea nas regiões de coluna lombar e fêmur proximal (absorciometria de raios-X de dupla energia-DEXA), difundido pela Organização Mundial de Saúde (OMS), foi o método de avaliação da densidade mineral óssea.

O exame periodontal foi obtido através de pesquisador especialista, calibrado e cego em relação a presença ou ausência de osteoporose. Como critério de classificação da doença periodontal foi utilizado o de Page \& Eke (2007) e Eke et al., (2012), mais utilizado internacionalmente.

Seguindo os critérios de diagnóstico Page \& Eke, para classificar e dizer se o indivíduo apresenta doença periodontal, o mesmo, deve apresentar pelo menos 2 sítios interproximais com NIC maior ou igual a $3 \mathrm{~mm}$ E pelo menos 2 sítios com PS maior ou igual $4 \mathrm{~mm}$ em dentes diferentes $\underline{\mathrm{OU}} 1$ sítio com profundidade de sondagem maior ou igual a 5 $\mathrm{mm}$. O sítio de PS maior ou igual a $4 \mathrm{~mm}$ tem que coincidir com um dos sítios do NIC.

Neste estudo, foi realizada a análise bivariada de co-variáveis para avaliar sua influência nos resultados. Dentre todas as variáveis, a presença da osteoporose, idade, escolaridade e fumo se mostraram como potenciais confundidoras, podendo influenciar a doença periodontal (Tabela 1)

Tabela 1- Análise das co-variáveis avaliadas para a Doença Periodontal, Feira de Santana - Ba

\begin{tabular}{lr|c|c|c}
\hline \multicolumn{5}{c}{ DOENÇA PERIODONTAL } \\
\hline \multirow{2}{*}{ OSTEOPOROSE } & NÃO & $43(14,2 \%)$ & $38(12,6 \%)$ & \multirow{2}{*}{ SIM } \\
& SIM & $86(28,5 \%)$ & $134(44,5 \%)$ & 0,03 \\
\hline \multirow{2}{*}{ IDADE } & $<60$ & $71(23,5 \%)$ & $56(18,6 \%)$ & $<0,01$ \\
& $>=60$ & $58(19,2 \%)$ & $116(38,5 \%)$ & \\
\hline \multirow{2}{*}{ ESCOLARIDADE } & $>4$ anos & $25(8,3 \%)$ & $16(5,3 \%)$ & \multirow{2}{*}{0,01} \\
& Até 4 anos & $104(34,5 \%)$ & $156(51,8 \%)$ & \\
\hline \multirow{2}{*}{ FUMO } & NUNCA & $95(31,5 \%)$ & $94(31,2 \%)$ & $<0,01$ \\
& FUMOU & $34(11,2 \%)$ & $78(25,9 \%)$ & \\
\hline
\end{tabular}


Fonte: prontuários das participantes do estudo

Sendo a osteoporose tratada com suplementação de cálcio por este ser um mineral importante na composição óssea, cruzou-se as informações do uso de suplementação de cálcio e doença periodontal, neste grupo de mulheres.

Segundo a classificação adotada, observa-se que das $172(57,1 \%)$ mulheres com DP, 45 (14,9\%) faziam uso de cálcio e 127 (42,1\%), não. Já nas mulheres sem DP, 26 (8,6\%) faziam uso de cálcio e 103 (34,2\%), não (Tabela 2).

Tabela 2- Associação entre a Doença Periodontal e o uso de Cálcio, segundo Page \& Eke (2007), Feira de Santana- Ba

\begin{tabular}{|c|c|c|c|c|}
\hline & \multicolumn{2}{|c|}{$\begin{array}{l}\text { Periodontite } \\
\text { PAGE e EKE }\end{array}$} & \multirow[b]{2}{*}{ Total } \\
\hline & & Não & Sim & \\
\hline \multirow{2}{*}{ Faz uso de cálcio } & Não & $103(34,2 \%)$ & $127(42,1 \%)$ & $230(76,4 \%)$ \\
\hline & Sim & $26(8,6 \%)$ & $45(14,9 \%)$ & $71(23,5 \%)$ \\
\hline \multicolumn{2}{|l|}{ Total } & $129(42,8 \%)$ & $172(57,1 \%)$ & $301(100 \%)$ \\
\hline
\end{tabular}

Fonte: prontuários das participantes do estudo

Nesse estudo, com base na avaliação do uso de cálcio e a presença de periodontite por Page e Eke, não houve diferença entre os grupos $(\mathrm{p}=0,224)$. Apesar de não ter havido significância estatística, a medida epidemiológica aponta uma associação positiva, com OR de 1,4 (Tabela 3).

Tabela 3- Análise das medidas epidemiológicas, Feira de Santana-Ba

Chi-Square Tests

\begin{tabular}{|l|r|r|r|r|r|}
\hline & \multicolumn{1}{|c|}{ Value } & df & \multicolumn{1}{c|}{$\begin{array}{c}\text { Asymp. Sig. } \\
\text { (2-sided) }\end{array}$} & $\begin{array}{c}\text { Exact Sig. (2- } \\
\text { sided) }\end{array}$ & $\begin{array}{c}\text { Exact Sig. (1- } \\
\text { sided) }\end{array}$ \\
\hline Pearson Chi-Square & $1,476^{a}$ & 1 &, 224 & & \\
Continuity Correction $^{b}$ & 1,162 & 1 &, 281 & & \\
Likelihood Ratio & 1,492 & 1 &, 222 &, 273 &, 140 \\
Fisher's Exact Test & 1,471 & 1 &, 225 & & \\
Linear-by-Linear & 301 & & & & \\
Association & & & & & \\
N of Valid Cases & 301 & & & \\
\hline
\end{tabular}

Fonte: prontuários das participantes do estudo

No ensaio clínico de Krall et al. ${ }^{9}$, o uso de suplementação de cálcio reduziu em $60 \%$ a perda dentária. Embora tenha se observado efeito protetor da suplementação de cálcio na retenção dentária (OR: 0,4; IC 95\%:0,2 -0,9), destaca-se como principal limitação do estudo o auto-relato da quantidade de dentes perdidos ao longo da investigação.

Dawson-hughes ${ }^{7}$ et al demonstraram que a suplementação da dieta com cálcio reduz a perda óssea em mulheres na pós-menopausa com baixo consumo desse mineral e o risco de fraturas em mulheres idosas quando associado com vitamina $\mathrm{D}^{8}$.

São poucos os estudos que avaliaram o efeito da suplementação de cálcio ${ }^{9,} 10,11$ sobre a condição bucal. Embora os seus efeitos benéficos sobre a perda óssea sistêmica e redução de risco de fraturas sejam reconhecidos ${ }^{12,13}$ seu potencial papel sobre a condição periodontal ainda não tem sido amplamente compreendido.

\section{CONSIDERAÇÕES FINAIS (ou Conclusão)}


Não houve diferença entre os grupos $(\mathrm{p}=0,224)$ deste estudo nas condições presentes. Apesar de não ter havido significância estatística, a medida epidemiológica aponta uma associação positiva. A realização de um análise multivariada é necessária para compreensão da relação entre o cálcio e doença periodontal levando em consideração o papel das variáveis confundidoras para conclusões mais consistentes.

\section{REFERÊNCIAS}

1 ROSETTI, E.; SAMPAIO, L.; ZUZA, E.; CIOCA, S.; TOLEDO, B. 2007. A Influência da Menopausa no Desenvolvimento da Doença Periodontal: Revisão de Literatura. Revista de Periodontia. 17(4): 20-23.

2 MORAES, T.G.; ARANTES, D.C.; NASCIMENTO, L.S.; CORREA, A.M. 2013. Prevalência de Doença Periodontal em Mulheres Menopausadas Atendidas na Fundação Santa Casa de Misericórdia do Pará. Rev. Sociedade Brasileira de Reprodução Humana. 28(2): 61-67.

3 PASSOS, J.; GOMES-FILHO, I.; VIANNA, M.; CRUZ, S.; FARIAS, T. 2010. Osteoporose e seus Efeitos na Condição Periodontal: Abordagem Teórica e Proposta de Modelo Conceitual. Revista Periodontia. 20(1): 38-47.

4 PALLOS, D.; CESCHIN, A.; VICTOR, G.A.; BULHÕES, R.C.; QUIRINO, M.R.S. 2006. Menopausa: Fator de Risco para Doença Periodontal? Rev. Bras. Ginecol. Obstet. 28(5):292297.

5 GOLUB, L.M.; PAYNE, J.B.; REINHARDT, R.A.; NIEMAN, G.J. 2006. Can systemic diseases co-induce (not just exacerbate) periodontitis? A hypothetical \&quot;two-hit\&quot; model. J Dent Res. 85:102-105.

6 LENER, U.H. 2006. Inflammation-induced bone remodeling in periodontal disease and the influence of post-menopausal osteoporosis. J Dent Res. 85:596- 607.

7 DAWSON-HUGHES, B.; DALLAL, G.E.; KRALL, E.A.; SADOWSKI, L.; SAHYOUN, N.; TANNENBAUM, S.A. 1990. Controlled trial of the effect of calcium supplementation on bone density in postmenopausal women. The New England Journal of Medicine. 323: 878883.

8 DAWSON-HUGHES, B.; HARRIS, S.S.; KRALL, E.A.; DALLAL, G.E. 1997. Effect of calcium and vitamin D supplementation on bone density in men and women 65 years of age or older. The New England Journal of Medicine. 337: 670-676.

9 KRALL, E.A.; WEHLER, C.; GARCIA, R.I.; HARRIS, S.S.; DAWSON-HUGHES, B. 2001. Calcium and vitamin D supplements reduce tooth loss in the elderly. Am J Med. 111:452-45.

10 GARCIA, M.N.; HILDEBOLT, C.F.; MILEY, D.D.; DIXON, D.A.; COUTURE, R.A; ANDERSON SPEARIE, C.L. et al. 2011. One-Year Effects of Vitamin D and Calcium Supplementation on Chronic Periodontitis. J Periodontol. 82:25-32.

11 MILEY, D.D.; GARCIA, M.N.; HILDEBOLT, C.F.; SHANNON, W.D.; COUTURE, R.A.; ANDERSON SPEARIE C.L. et al. 2009. Cross-sectional study of vitamin D and calcium supplementation effects on chronic periodontitis.J Periodontol. 80:1433-9.

12 BOONEN, S.; VANDERSCHUEREN, D.; HAENTJENS, P.; LIPS, P. 2006. Calcium and vitamin $\mathrm{D}$ in the prevention and treatment of osteoporosis - a clinical update. J Intern Med. 259(6):539-552.

13 JACKSON, R.D.; LACROIX, A.Z.; GASS M. et al. 2006. Calcium plus vitamin D supplementation and the risk of fractures. New Eng J Med. 354(7):669-683. 